\title{
Differentially expressed genes associated with TMPRSS2- ERG molecular subtype of prostate cancer
}

\author{
Anastasiya Andreevna Kobelyatskaya \\ Laboratory of Postgenomic Research \\ EIMB RAS \\ Moscow, Russia \\ kaa.chel@mail.ru
}

Anna Victorovna Kudryavtseva

Laboratory of Postgenomic Research EIMB RAS

Moscow, Russia

rhizamoeba@mail.ru

\author{
Elena Anatolevna Pudova \\ Laboratory of Postgenomic Research \\ EIMB RAS \\ Moscow, Russia \\ pudova_elena@inbox.ru \\ Kirill Mikhailovich Nyushko \\ Urological department \\ FSBI NMRRC \\ Moscow, Russia \\ kirandja@yandex.ru
}

\author{
George Sergeevich Krasnov \\ Laboratory of Postgenomic Research \\ EIMB RAS \\ Moscow, Russia \\ gskrasnov@mail.ru \\ Boris Yakovlevich Alekseev \\ Urological department \\ FSBI NMRRC \\ Moscow, Russia \\ mnioi@mail.ru
}

\begin{abstract}
Prostate cancer (PC) is one of the most common and socially significant oncological diseases in men. This study examined the transcriptome profile of the most common molecular genetic subtype of prostate cancer, TMPRSS2-ERG. As a result of bioinformatics analysis conducted on the basis of The Cancer Genome Atlas project data, 115 differentially expressed genes were identified for this study group, and in particular, the most over-expressing genes were identified: ALOX15, CACNA1D, EML6, HLA-DMB, NKAIN1, OGDHL, PLA1A, SYT13. Enrichment pathways analysis showed that these genes are participants in important oncologically significant pathways, which emphasizes the association of this molecular subtype with an unfavorable prognosis for prostate cancer.
\end{abstract}

Keywords - TMPRSS2-ERG, TCGA, prostate cancer, sequencing

\section{Introduction}

Prostate cancer (PC) is one of the most common and socially significant oncological diseases in men. At the molecular genetic level, prostate cancer can be divided into seven subtypes, the most common among them being the subtype characterized by the presence of the chimeric transcript TMPRSS2-ERG (up to $45 \%$ of cases) $[1,2]$. This transcript is the result of translocation between exon 1 of TMPRSS2 gene and exon 4 of ERG gene [2, 3]. According to the results of numerous studies, the presence of the TMPRSS2-ERG transcript is most often considered an important predictor of unfavorable prognosis $[1,2,4]$. The aim of our study is to investigate the characteristics of the transcriptome profile of this molecular subtype, which can serve as a basis for understanding the mechanisms of progression in prostate cancer and help in the search for informative prognostic markers.

\section{Methods}

The study included PC samples RNA-Seq data of The Cancer Genome Atlas project. The cohort was divided into two groups: tumors with TMPRSS2-ERG fusion transcript (88 cases) and TMPRSS2-ERG-free tumors (117 cases). Samples have appropriate $E R G$ expression signature. Differential expression analysis was performed in statistical environment $\mathrm{R}$ using the Edge $\mathrm{R}$ package was used. The Mann-Whitney test, Exact test and quasi-likelihood method (QLF) were used for statistical analysis. To exclude false positive results FDR method was used. Enrichment pathways analysis was performed using clusterProfiler and ReactomePA packages. The results were considered statistically significant with a QLF p-value $<0.05$.

\section{Results}

We found out at list 115 differentially expressed genes between the studied groups (fig.1 - Differentially expressed genes TMPRSS2-ERG molecular subtype PC). Among those next genes are of most interest with fold-expression level more than 4 for TMPRSS2-ERG-positive group: ALOX15, CACNA1D, EML6, HLA-DMB, NKAIN1, OGDHL, PLA1A, SYT13. The following genes showing overexpression in TMPRSS2-ERG-positive group of samples are marked: ALOX15, CACNA1D, EML6, HLA-DMB, NKAIN1, OGDHL, PLA1A, SYT13. According to the results of enrichment pathways analysis, these genes are participants in the following cancer-significant pathways in the KEGG database associated with the progression: Arachidonic acid metabolism (hsa00590), Focal adhesion (hsa04510), Mucin type O-glycan biosynthesis (hsa00512), Notch signaling pathway (hsa04330), PI3K-Akt signaling pathway (hsa04151), Prostate cancer (hsa05215), Sphingolipid metabolism (hsa00600). Thus, the results underscore the potential association with an unfavorable prognosis in the group of samples belonging to the molecular subtype TMPRSS2-ERG.

\section{ACKNOWLEDGMENT}

This work was funded by the Russian Science Foundation grant no. 18-75-10127. This work was performed using the equipment of EIMB RAS "Genome" center (http://www.eimb.ru/ru1/ckp/ccu_genome_c.php).

\section{REFERENCES}

[1] Arora K., Barbieri C.E. (2018) Molecular Subtypes of Prostate Cancer. Curr Oncol Rep. 20(8):58. doi: 10.1007/s1 1912-018-0707-9.

[2] Cancer Genome Atlas Research Network. (2015) The molecular taxonomy of primary prostate cancer. Cell. 163(4):1011-25. doi: 10.1016/j.cell.2015.10.02.

[3] Krumbholz M., Agaimy A., Stoehr R., Burger M., Wach S., Taubert H., Wullich B., Hartmann A., Metzler M. (2019) Molecular Composition of Genomic TMPRSS2-ERG Rearrangements in Prostate Cancer. Dis Markers. 2019:5085373. doi: 10.1155/2019/5085373.

[4] Wang Z., Wang Y., Zhang J., Hu Q., Zhi F., Zhang S., Mao D., Zhang Y., Liang H. (2017) Significance of the TMPRSS2:ERG gene fusion in prostate cancer. Mol Med Rep. 16(4):5450-5458. doi: $10.3892 / \mathrm{mmr} .2017 .7281$. 